\title{
RADIAL FUNCTIONS ON COMPACT SUPPORT
}

\author{
by M. D. BUHMANN
}

(Received 22nd November 1995)

\begin{abstract}
In this paper, radial basis functions that are compactly supported and give rise to positive definite interpolation matrices for scattered data are discussed. They are related to the well-known thin plate spline radial functions which are highly useful in applications for gridfree approximation methods. Also, encouraging approximation results for the compactly supported radial functions are shown.
\end{abstract}

1991 Mathematics subject classification: 41A05, 41A25, 41A63, 42A82, 65D05, 65D07.

\section{Introduction}

The theme of this work is the construction of a new class of radial basis functions. Their special features are compact support and good approximation properties when they are used for interpolation (or other approximations). Radial basis functions and their applications have been comprehensively reviewed in several recent papers $([4,6,13]$, to name just three). Therefore we will not dwell much on explaining radial basis functions here. Be it sufficient to mention that radial basis function schemes are multivariate approximations from linear spaces generated by translates of the form $\phi\left(\cdot-x_{j}\right)=\tilde{\phi}\left(\left\|\cdot-x_{j}\right\|\right): \mathbb{R}^{n} \rightarrow \mathbb{R}$, where $\tilde{\phi}: \mathbb{R}_{+} \rightarrow \mathbb{R}$ is the radial basis function, $\|\cdot\|: \mathbb{R}^{n} \rightarrow \mathbb{R}_{+}$is the Euclidean norm (the modulus function in one dimension) and $x_{j} \in \mathbb{R}^{n}$ are prescribed points (called centres or sometimes knots) in the underlying space. The usual way to approximate by these functions is through interpolation at the centres, giving rise to the question of the invertibility of the interpolation matrix $\left\{\phi\left(x_{i}-x_{j}\right)\right\}_{i, j}$ that we shall address, among other issues, in this article. We also call $\phi$ by the name of radial basis function.

In this paper, we will study approximation spaces generated by a novel type of compactly supported radial basis functions $\phi$, in contrast to most of the work reviewed, for instance, in [4], where globally supported functions are taken. The advantage of compact support for radial function methods, as studied in the related papers with different approaches [14] and [19], is, on one hand, that the linear systems resulting from interpolation from these spaces are easy to solve, and, on the other hand, that the resulting interpolants can be evaluated very fast. Frequent evaluation is, indeed, often desired in applications. An example for this is the visual rendering of two-dimensional functions. The various well-known globally supported radial functions, such as thin plate splines and multiquadrics, have many good 
(especially approximation-) properties, but they need much extra attention for solving the linear systems of interpolation efficiently, because the interpolation matrices are full and can be badly conditioned.

Further, radial basis functions with compact support are especially interesting in the context of solving partial differential equations, for example with boundary element methods, because they can be integrated easily by numerical quadrature since the integrals involved are finite. On the other hand, several of the globally supported radial functions are fundamental solutions of elliptic operators. As such they can even be integrated analytically. For example, the thin plate spline function $\tilde{\phi}(r)=r^{2} \log r$ has in two dimensions the property

$$
16 r^{2} \log r=\Delta\left(r^{4} \log r-\frac{1}{2} r^{4}\right) .
$$

Here, $r=\|x\|$ and $\Delta$ is the Laplace operator. Our new functions unite these advantages because they are in many cases piecewise fundamental solution functions with finite support. Therefore they are useful for creating the trial spaces for such numerical methods for differential equations. A further reason for this interest from the differential equation point of view is the possibility to perform gridfree approximations for finite elements with radial basis functions (Schwab, private communication). The assumption of gridded centres just for the convergence analysis in this paper is no contradiction to this statement, since its precise purpose is the identification of the general approximational efficacy of the radial function spaces.

To comment further on the work of the two papers mentioned before, $\mathrm{Wu}$ [19] shows how to find compactly supported radial basis functions $\phi$ which are positive definite on ambient spaces of fixed dimension (degree and admitted dimension are interrelated). Viewed as functions $\tilde{\phi}$, they are piecewise polynomial functions which have nonnegative, nontrivial Fourier transform, when transformed as functions $\phi$ in $\mathbb{R}^{n}$. This means, by a famous theorem of Bochner, that the interpolation matrices for interpolation at the centres are positive definite if the centres are distinct and lie in $\mathbb{R}^{n}$. Therefore they are called positive definite functions. For his purpose, Wu makes essential use of nonnegativity of the Fourier transform of $\phi$, if $\tilde{\phi}$ is taken from a set of certain multiply monotone functions, see also $[11,3]$. Specifically, he creates positive definite radial functions $\phi$ in one dimension first, and then uses a certain differentiation operator, applied to $\tilde{\phi}$, to lift these univariate functions that give rise to positive definite interpolation matrices to higher dimensions (and lower smoothness). This shows how degree, smoothness and dimension are related. It is a fundamental new contribution in that article to identify differentiation as the link between positive definite radial functions in spaces whose dimensions differ. In [14] radially symmetric functions with positive Fourier transform are obtained by convolving characteristic functions of balls with themselves or by using tensor product splines and radializing the result, mainly in two and three dimensions (the former are called "Euclid's hats"). Finally, Wendland [17] finds functions that are closely related to Wu's radial basis functions but proves that these have minimal polynomial degree, given the support size and the smoothness. 
In this paper, we deal first, in the next section, with the creation of a new class of radial functions that have compact support and positive Fourier transform, thus giving rise to interpolation matrices which are positive definite for distinct centres. In the third section, convergence orders for these radial functions which resemble the wellknown thin plate splines, see [8], are discussed. The approximation results obtained rely on either implementing a scaling of the radial function that is different from the spacing of the centres, or letting the "degree" of the radial function tend to infinity. In the latter case, which is studied in the final section, a spectral convergence result is obtained. At the time of writing and when this work was presented at conferences at Cancun, Montecatini and Oberwolfach in 1995, it appeared to be the first specific discussion of approximation orders of compactly supported radial functions. The author apologizes if this is an oversight.

\section{The radial basis functions}

We require compactly supported radial basis functions with positive Fourier transform, and pursue an altogether different tack than [19] to obtain functions related to the well-known thin plate splines. We want to apply the results in [12] about positive integrals of Bessel functions. Therefore the radial basis functions we seek are generally represented by integrals

$$
\phi(x)=\int_{0}^{\infty}\left(1-\|x\|^{2} / \beta\right)_{+}^{\lambda} g(\beta) d \beta, \quad x \in \mathbb{R}^{n},
$$

where $g \in C_{c}\left(\mathbb{R}_{+}\right)$, the space of compactly supported continuous functions on $\mathbb{R}_{+}$. Thus the result is clearly of compact support. Similar radial basis functions that are related to multiply monotone functions and their Williamson representation [18] were already considered in $[11,3]$, but they were not of compact support there, i.e., the weight $g$ was not compactly supported. We require $\hat{\phi}>0$ everywhere. The compact support, and therefore the integrability of $\phi$ and the existence of a continuous (in fact: analytic) Fourier transform are guaranteed by the compact support of $g$ in (2.1). Our ansatz yields the following Fourier transform which is amenable to an application of the work in [12]. In the sequel, $C$ denotes a generic positive constant whose exact value is immaterial.

$$
\begin{aligned}
\hat{\phi}(x) & =C\|x\|^{1-n / 2} \int_{0}^{\infty} \int_{0}^{\infty}\left(1-s^{2} / \beta\right)_{+}^{\lambda} s^{n / 2} J_{n / 2-1}(s\|x\|) d s g(\beta) d \beta \\
& =C\|x\|^{-\lambda-n / 2} \int_{0}^{\infty} J_{\lambda+n / 2}(\|x\| \sqrt{\beta}) \beta^{n / 4-\lambda / 2} g(\beta) d \beta \\
& =C\|x\|^{-n-2} \int_{0}^{\infty} J_{\lambda+n / 2}(t) t^{n / 2-\lambda+1} g\left(t^{2}\|x\|^{-2}\right) d t \\
& =C\|x\|^{-n-2-2 \mu e} \int_{0}^{\infty} J_{\lambda+n / 2}(t) r^{n / 2-\lambda+1}\left(\|x\|^{2 \mu}-t^{2 \mu}\right)_{+}^{e} d t .
\end{aligned}
$$


Here we have taken $g(\beta)=\left(1-\beta^{\mu}\right)_{+}^{e}$. The letter $J$ denotes the Bessel functions [1, p. 358] of the order according to the index. This Fourier transform is positive by [12, Corollary 2.4, using items (i), (ii) and (vi)], and the strict inequalities (1.1) and (1.5) in [9] if $0<\mu \leqslant \frac{1}{2} \leqslant \varrho$ and

$$
n=1, \quad \lambda \geqslant \frac{1}{2}
$$

or

$$
n \geqslant 2 \text { and } \lambda \geqslant \frac{1}{2}(n-1)
$$

An example is $\mu=\frac{1}{2}$ and $\varrho=1$, and we shall further on always take $\mu=\frac{1}{2}$, whereas higher powers $\varrho$ are admitted. As a simplifying assumption we suppose $\lambda, \varrho \in \mathbb{N}$, although that is not necessary and entails no limitation to the generality of the results. We have thus proved

Theorem 1. For $g(\beta)=\left(1-\beta^{\mu}\right)_{+}^{e}, 0<\mu \leqslant \frac{1}{2} \leqslant \varrho$, the Fourier transform of $(2.1)$ is everywhere well-defined and positive whenever $\lambda$ satisfies one of the two displayed conditions above.

\section{Properties of the radial basis functions}

We study some of the approximation properties of our newly constructed radial functions in this section. Notably, we discuss in several dimensions and for equally spaced centres which approximation properties are obtainable, if only the scaling is chosen judiciously. For this, the scaling of the radial function has to tend to zero at a different rate than the spacing of the centres. In practice this can be useful when scattered centres are distributed with unequal density, so that the scaling of the basis function should be locally adapted. Compact support radial basis functions seem to be very amenable to this case.

The first interesting fact we observe is that, if $\lambda$ and $\varrho$ are integral, $\mu=\frac{1}{2}$ and $\varrho \geqslant \lambda$,

$$
\begin{aligned}
\phi(x) & =\int_{0}^{\infty}\left(1-\|x\|^{2} / \beta\right)_{+}^{\lambda}(1-\sqrt{\beta})_{+}^{e} d \beta \\
& =\int_{\|\left. x\right|^{2}}^{1}\left(1-\|x\|^{2} / \beta\right)^{\lambda}(1-\sqrt{\beta})^{e} d \beta \\
& =p(\|x\|)+q\left(\|x\|^{2}\right) \log \|x\|,
\end{aligned}
$$

for $\|x\| \leqslant 1$, where $p$ and $q$ are polynomials, the latter of degree $\lambda$, and $p(1)=q(0)=0$.

Since $\phi$ is of compact support and not a piecewise polynomial, its multi-integer translates, failing to be a partition of unity, cannot generate a space that contains nontrivial polynomials, as can also be seen from the Strang and Fix conditions for 
polynomial recovery by translates of a function. Hence, by a theorem of Buhmann, [4, p. 44], approximation orders of cardinal interpolation with these functions and the usual, stationary scaling cannot be expected. (Other, much more general theorems delivering this result are available, but we require no more than a special case of that assertion in the present work.) The stationary scaling is the one which keeps the relative support of the radial function with respect to the shrinking gridsize constant.

Hence we consider the approximation properties of scales of such radial functions that are made so as to enlarge the support of the scaled function relative to the spacing of the translates (i.e., the gridsize $h>0$ ). This growth should be slow, by $h^{-\gamma+1}$, say, where $0 \ll \gamma<1$. We shall see that faster growth gives better approximation orders, but as we normally intend to keep the support small, it should be as slow as a reasonable approximation order allows. Therefore, whenever $\lambda$ and $\varrho$ are integral, we consider here properties of spaces generated by

$$
q\left(\left\|h^{-\gamma}(x-j h)\right\|^{2}\right) \log \left\|h^{-\gamma}(x-j h)\right\|+p\left(\left\|h^{-\gamma}(x-j h)\right\|\right), \quad j \in \mathbb{Z}^{n}, h>0 .
$$

An example is $\lambda=\varrho=1, \mu=\frac{1}{2}$, which gives $q(\tilde{x})=2 \tilde{x}, p(\tilde{x})=\frac{1}{3}+\tilde{x}^{2}-\frac{4}{3} \tilde{x}^{3}$, i.e.,

$$
\phi(x)= \begin{cases}\frac{1}{3}+\|x\|^{2}-\frac{4}{3}\|x\|^{3}+2\|x\|^{2} \log \|x\| & \text { if } 0 \leqslant\|x\| \leqslant 1, \\ 0 & \text { otherwise. }\end{cases}
$$

Another example is $\lambda=1, \varrho=4, \mu=\frac{1}{2}$, which gives

$$
\phi(x)= \begin{cases}\frac{1}{15}+\frac{19}{6}\|x\|^{2}-\frac{16}{3}\|x\|^{3}+3\|x\|^{4}-\frac{16}{15}\|x\|^{5}+\frac{1}{6}\|x\|^{6}+2\|x\|^{2} \log \|x\| & \text { if } 0 \leqslant\|x\| \leqslant 1, \\ 0 & \text { otherwise. }\end{cases}
$$

These $\phi \in C^{\prime}(\mathbb{R})$. Indeed this is the smoothness that can be obtained due to the nature of the Euclidean norm inside the truncated power in the definition of our radial functions. The idea of studying scales of radial functions not proportional to the gridsize occurs already in [5], where discrete least-squares approximations from spaces of radial basis functions scaled by another quantity than the gridspacing were analysed in much detail (see also [2]).

These radial functions have the additional advantage that they can be integrated easily against piecewise polynomials. This is important when, for example, inner products of the radial functions as trial functions against splines as test functions are required for the numerical solution of differential equations (by finite element or similar methods). As a simple example just for illustration, we consider their convolution with constant B-splines, i.e., characteristic functions of the shifted unit interval. This preserves their general form, but increases the polynomial degree. For example, using (3.2), 


$$
\int_{\max [x, 0]}^{\min [x+1,1]} \frac{1}{3}+t^{2}-\frac{4}{3} t^{3}+2 t^{2} \log t d t= \begin{cases}\text { polynomial }+\frac{2}{3}(x+1)^{3} \log (x+1) & \text { if }-1 \leqslant x \leqslant 0 \\ \text { polynomial }-\frac{2}{3} x^{3} \log x & \text { if } 0 \leqslant x \leqslant 1 \\ 0 & \text { otherwise }\end{cases}
$$

where $x \in \mathbb{R}$.

We continue with the convergence analysis for the radial functions of Theorem 1. Those will be the only ones we deal with. As alluded to above, we must give attention to the scaling of the radial function which is not going to be the same as the scaling of the grid of centres we use. The latter we denote by $h>0$, the former from now on by $\delta>0$. This generalizes (3.1), where $\delta=h^{7}$ was used. Hence we consider $\phi_{\delta}=\phi\left(\frac{\dot{z}}{\bar{b}}\right)$, the radial function $\phi$ scaled in the described way. As in [13], we call the linear space of tempered distributions $f$ that fulfill

$$
\|f\|_{\phi_{\delta}}^{2}:=\frac{1}{(2 \pi)^{n}} \int_{\mathrm{R}^{n}} \frac{|\hat{f}(x)|^{2}}{\hat{\phi}_{\delta}(x)} d x<\infty
$$

the native space $\mathcal{F}_{\phi_{\delta}}$ of the (scaled) radial function. Spaces of this type are first considered in the important work [8] and several later continuations of the seminal work by Golomb and Weinberger [10]. We also consider $\mathcal{F}_{\phi}=\mathcal{F}_{\phi_{1}}$. Note that this norm is well-defined since $\phi_{\delta}$ is absolutely integrable due to its limited support, so the Fourier transform exists, and we may divide by $\hat{\phi}_{\delta}$ because it is positive by construction. The theorem we are going to prove is as follows. In its statement we use the familiar notation $D^{-n / 2-1} L^{2}\left(\mathbb{R}^{n}\right)$ for $\mathcal{F}_{\psi}$, where $\hat{\psi}=\|\cdot\|^{-n-2}$. We note that due to the Sobolev embedding theorem $D^{-n / 2-1} L^{2}\left(\mathbb{R}^{n}\right) \subset C\left(\mathbb{R}^{n}\right)$ for all $n \in \mathbb{N}$. Moreover, $\delta(\cdot)$ is the well-known delta-distribution or, in case of a discrete argument, the Kronecker delta-function.

Theorem 2. Let $f \in L^{2}\left(\mathbb{R}^{n}\right) \cap D^{-n / 2-1} L^{2}\left(\mathbb{R}^{n}\right)$. Suppose also $\varrho \geqslant \frac{1}{2} n+3+2 \mu-\lambda$. Then there is a cardinal interpolant for $h>0$

$$
s(x)=\sum_{j \in \mathbf{Z}^{n}} f(j h) u_{j h}^{*}(x), \quad x \in \mathbb{R}^{n},
$$

from the linear space generated by $h \mathbb{Z}^{n}$-translates of $\phi_{\delta}$, where the Lagrange functions are

$$
u_{j h}^{*}(x)=\sum_{k \in \mathbb{Z}^{n}} c_{k, h, \delta} \phi_{\delta}(x-j h-k h), \quad x \in \mathbb{R}^{n}
$$

and the Lagrange conditions

$$
u_{j h}^{*}(k h)=\underline{\delta}(j-k), \quad j, k \in \mathbb{Z}^{n},
$$


hold. This interpolant satisfies the uniform error estimate

$$
\|f-s\|_{\infty} \leqslant C h \delta^{-1}\left(\delta^{-n / 2}\|f\|_{2}+\delta\|f\|_{\psi}\right), \quad h>0, h / \delta \in(0,1) .
$$

In (3.8), C does not depend on either $h, \delta,\|f\|_{2}$ or $\|f\|_{\psi}$.

Proof. The existence, uniqueness and other properties of cardinal functions (3.6) for basis functions of positive Fourier transform have been extensively discussed in the literature. What interests us here is the existence of the cardinal functions. They can be expressed in terms of their Fourier transform. According to [7, eqn. (2.1), p. 322], for instance,

$$
\hat{u}_{0}^{*}(x)=\frac{h^{n} \hat{\phi}(x)}{\sum_{j \in \mathbb{Z}} \hat{\phi}\left(x-\frac{2 \pi}{h}\right)}, \quad x \in \mathbb{R}^{n},
$$

when $\delta=1$. This Fourier transform is continuous. It is absolutely integrable too, since it decays like $\hat{u}_{0}^{*}(x)=O\left(\|x\|^{-n-2}\right)$ for large argument as $\hat{\phi}(x)$ does. However, we postpone the proof of the latter fact to the proof of the next proposition, because we shall require even more detailed properties of $\hat{\phi}$. Nonetheless, we conclude already at this point that the Fourier transform (3.9) defines $u_{0}^{*}$ which is at a minimum continuous. The other $u_{j h}^{*}$ are just translates $u_{j h}^{*}=u_{0}^{*}(\cdot-j h)$ thereof. The $u_{0}^{*}$ decays faster than any negative power. This can be seen from the fact that its Fourier transform (3.9) is infinitely often continuously differentiable, since the compact support of $\phi$ implies analyticity of $\hat{\phi}$. Therefore all tempered distributions $f$ that are also continuous functions are admitted into the interpolant (3.5), and, of course, the hypotheses about $f$ in the statement of the theorem restrict it to that class. The cardinal functions for $\delta \neq 1$ are found by simply scaling $\hat{\phi}$ in (3.9) by $\delta$.

For all radial functions with compact support and a Fourier transform that is positive, the following error estimate holds. This is going to be the basis of the rest of our proof. It applies to any $f$ for which an interpolant $s$ on $h \mathbb{Z}^{n}$ by the radial function in question is well-defined. (We admit in the estimate $\|f\|_{\phi_{b}}=\infty$, so it remains true if $f \notin \mathcal{F}_{\phi_{\delta}}$, but we shall see later that $\|f\|_{\phi_{\delta}}<\infty$ under the hypothesis of the theorem.) The inequality is

$$
|f(x)-s(x)| \leqslant\|f\|_{\phi_{\delta}} \sqrt{F_{\phi}\left(\frac{h}{\delta}\right)},
$$

as shown in [13, eqn. (4.8)]. Here, $F_{\phi}$ is the so-called power functional

$$
F_{\phi}(\tilde{h})=\sup _{|x|_{\infty}<h}\left|\phi(0)-2 \sum_{j \in \mathbb{Z}^{*}} \underline{u}_{j \tilde{h}}^{*}(x) \phi(x-j \tilde{h})+\sum_{k \in \mathbb{Z}^{n}} \sum_{j \in \mathbb{Z}^{n}} \underline{u}_{k h}^{*}(x) \underline{u}_{j h}^{*}(x) \phi(k \tilde{h}-j \tilde{h})\right|, \quad \tilde{h}=\frac{h}{\delta}>0 .
$$

In this power functional, $\underline{u}_{j h}^{*}$ can be a priori any function for which the sums are well- 
defined, but if it satisfies the Lagrange conditions, the right-hand side of (3.10) is minimal because this corresponds to an exact expression of the error functional. A convergence result can be obtained by estimating the two factors that appear on the right-hand side of (3.10). Of course the estimate must be such that is tends to zero, when both $\delta$ and $h$ go to 0 , albeit at different rates. We begin with the second one.

It is well-known that the aforementioned power functional in (3.10) is minimized if $\underline{u}_{j h}^{*}$ are the Lagrange or cardinal functions $u_{j h}^{*}$ from the radial function space spanned by the $h \mathbb{Z}^{n}$-translates of $\phi([13]$, for example; other references are available). We assume that choice from now on.

Using these facts we can prove the following first result on our way to bounding (3.10) uniformly from above by a decaying term.

Proposition 3. The quantity in (3.10)

$$
\sqrt{F_{\phi}\left(\frac{h}{\delta}\right)}
$$

can be uniformly bounded by a fixed multiple of $h / \delta$ so long as $h / \delta \in(0,1)$. The multiplier is independent of $\delta$ and $h$, but depends on $\phi$ and $n$.

Proof. We need an auxiliary result to prepare for the proof. A straightforward generalization of Proposition 6 in [7, p. 328] to general radial functions with positive Fourier transform (not multiquadric radial functions) and, for our purpose, compact support, gives

Lemma 4. For the radial functions constructed in Theorem 1, it is true that the cardinal interpolant to the radial function itself recovers the latter:

$$
\sum_{k \in \mathbb{Z}^{n}} \phi(k h-j h) u_{k h}^{*}(x)=\phi(x-j h), \quad x \in \mathbb{R}^{n}, \quad j \in \mathbb{Z}^{n}, \quad h>0
$$

What we are using here, and what is proved in the proposition in the paper referred to, is the projection property of radial function interpolants on the scaled integer grid. As the proof of this fact for our compactly supported radial functions with positive Fourier transform is even easier than the proof for the multiquadric function, we omit it.

Therefore the power functional can be reexpressed by

$$
F_{\phi}(h)=\sup _{\|x\|_{\infty} \leqslant h}\left|\phi(0)-\sum_{j \in \mathbf{Z}^{n}} u_{j h}^{*}(x) \phi(x-j h)\right| .
$$

In the notation for this equation we have replaced $\frac{h}{\delta}$ by $h$ for reasons of notational convenience. The back substitution at the end of the proof of the proposition will be trivial. 
An application of the Poisson summation formula, [15, p. 252], transforms the expression inside the modulus signs in (3.11) into a multiple of

$$
h^{-2 n} \int_{\mathbf{R}^{n}} \sum_{k \in \mathbb{Z}^{n}}(1-\exp (2 \pi i k x / h)) \hat{\phi}\left(\frac{t-2 \pi k}{h}\right) \hat{u}_{0}^{*}\left(\frac{t}{h}\right) d t .
$$

Using the form of $u_{0}^{* \prime}$ 's Fourier transform (3.9), we get that this is the same as

$$
\int_{\mathrm{R}^{n}} \sum_{k \in \mathbb{Z}^{n} \backslash(0)}(1-\exp (2 \pi i k x / h)) \hat{\phi}\left(t-\frac{2 \pi k}{h}\right) \hat{\phi}(t) \frac{d t}{\sum_{\ell \in Z^{n}} \hat{\phi}\left(t-\frac{2 \pi l}{h}\right)} .
$$

We require an auxiliary result in order to bound this expression. The following lemma which is a consequence of Lemma 7 in [3] shows that we get $\hat{\phi}(t) \sim(1+\|t\|)^{-n-2}$.

Lemma 5. Suppose we are given $q \in \mathbb{N}$ and $\xi \in C^{q}\left(\mathbb{R}_{>0}\right)$, and suppose that for $J \in \mathbb{N}$ we have the asymptotic expansion

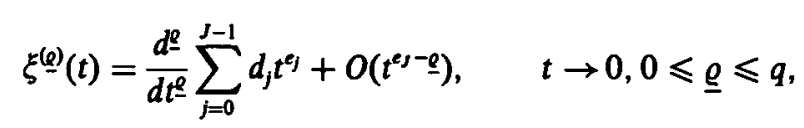

with real numbers $d_{f}$ and reals $e_{0}<e_{1}<\cdots<e_{j}$, where $e_{0}>-v-1$ and where $v=\lambda+\frac{1}{2} n, \lambda>0, q \geqslant 2 e_{J}+v+2$. Let

$$
\hat{\phi}(r)=r^{-0-1 / 2} \int_{0}^{\infty} \underline{h}(r \beta) f(\beta) d \beta, \quad r>0,
$$

where $\left\{\underline{h}(t)=J_{0}(t) \sqrt{ } t \mid t>0\right\}$ and where

$$
f(\beta)=2^{0+1} \beta^{0+1 / 2} \xi\left(\beta^{2}\right), \quad \beta>0,
$$

be well-defined for every positive $r$. Then it satisfies for any $0<\varepsilon<2 \min \left(e_{0}+v+1\right.$, $\left.e_{J}-e_{J-1}, \frac{1}{2} v\right)$ the asymptotic expansion

$$
\hat{\phi}(r)=\sum_{j=0}^{J-1} \frac{\Gamma\left(v+e_{j}+1\right) d_{j}}{\Gamma\left(-e_{j}\right)}\left(\frac{1}{2} r\right)^{-2 e_{j}-2 v-2}+O\left(r^{-2 e j-2 v-2+\theta}\right), \quad r \rightarrow \infty .
$$

For our purpose, we invoke Lemma 5 with $q=\varrho-1 \geqslant 2 e_{1}+v+2, J=1, a_{0}=1$, $e_{0}=-\lambda, e_{1}=\mu-\lambda$ and $\xi(\beta)$ a constant multiple of the compactly supported function $g(\beta) \beta^{-\lambda}$. It follows that $\hat{\phi}(t)=O\left(\|t\|^{-n-2}\right)$ for large $\|t\|$. Moreover, we see that the Fourier transform can be bounded below for sufficiently large argument by a small constant multiple of $\|\cdot\|^{-n-2}$ : Indeed, a consequence of (3.13), that is,

$$
\hat{\phi}(r)=C r^{-n-2}+O\left(r^{-n-2-\varepsilon}\right), \quad r \rightarrow \infty,
$$


for a small positive $\varepsilon$ implies for large enough $r$

$$
\hat{\phi}(r) \geqslant \frac{1}{2} C r^{-n-2}, \quad r \rightarrow \infty .
$$

This means that $\hat{\phi}(t) \sim(1+\|t\|)^{-n-2}$, because we already know that $\hat{\phi}>0$ always. The asymptotic estimate will help us with the rest of the proof.

To continue, we remark that (3.12) can be bounded above by a constant multiple of

$$
\begin{aligned}
\int_{\mathbf{R}^{n}} \sum_{k \in \mathbb{Z}^{n} \backslash\{0\}}(1+ & \left.\left\|t-\frac{2 \pi k}{h}\right\|\right)^{-n-2}(1+\|t\|)^{-n-2} \frac{d t}{\sum_{\ell \in \mathbb{Z}^{n}}\left(1+\left\|t-\frac{2 \pi \ell}{h}\right\|\right)^{-n-2}} \\
& \leqslant h^{2} \int_{\mathbb{R}^{n}} \sum_{k \in \mathbb{Z}^{n} \backslash\{0\}}(h+\|t-2 \pi k\|)^{-n-2}(h+\|t\|)^{-n-2} \frac{d t}{\sum_{\ell \in \mathbb{Z}^{n}}(h+\|t-2 \pi \ell\|)^{-n-2}} .
\end{aligned}
$$

The right-hand side expression is for $h<1$ at most

$$
\begin{aligned}
h^{2} \int_{\mathbf{R}^{n}} \sum_{k \in \mathbb{Z}^{n} \backslash\{0\}}(h+\|t-2 \pi k\|)^{-n-2}(h+\|t\|)^{-n-2} \frac{d t}{\sum_{\ell \in \mathbb{Z}^{n}}(1+\|t-2 \pi \ell\|)^{-n-2}} \\
\leqslant C h^{2} \sum_{k \in Z^{n} \backslash\{0\}}(h+\|2 \pi k\|)^{-n-2} \leqslant C h^{2} \sum_{k \in \mathbb{Z}^{n} \backslash\{0\}}\|2 \pi k\|^{-n-2}=O\left(h^{2}\right),
\end{aligned}
$$

as required.

According to (3.10) we are now only left with bounding $\|f\|_{\phi_{s}}$ uniformly in order to prove the convergence result. There is a small constant $C$ such that

$$
\hat{\phi}(\delta x) \geqslant C \begin{cases}\delta^{-n-2}\|x\|^{-n-2} & \text { if } C\|x\| \geqslant \delta^{-1} \\ 1 & \text { if } C\|x\| \leqslant \delta^{-1}\end{cases}
$$

Therefore we can use the dominated convergence theorem to estimate

$$
\begin{aligned}
\delta^{n}(2 \pi)^{n}\|f\|_{\phi_{b}}^{2} & =\int_{\mathbf{R}^{n}} \frac{|\hat{f}(x)|^{2} d x}{\hat{\phi}(\delta x)} \\
& \leqslant C \int_{\mathbf{R}^{n}}|\hat{f}(x)|^{2}+\delta^{n+2}\|x\|^{n+2}|\hat{f}(x)|^{2} d x=C\|f\|_{2}^{2}+C \delta^{n+2}\|f\|_{\psi}^{2} .
\end{aligned}
$$

This, in connection with the previous proposition and our assumptions about $f$ in the statement of the theorem, proves the assertion.

An application of the theorem we just proved shows that, if for instance $h=O\left(\delta^{3 n / 2+3}\right)$, then we get convergence order $O\left(h^{2 / 3}\right)$ for the approximant in any 
dimension. Faster decay of $h$ relative to $\delta$ results in higher rates of approximation, and we might also increase $\delta$ instead of letting it tend to zero. In other words, the support size is in competition with the accuracy, and the approximation order stated in Theorem 2 cannot, incidentally, match the orders obtainable with the globally supported radial functions, e.g., reviewed in [4]. In fact, $O(h)$ is the best obtainable order (for $\delta=1$ ) if we want to keep $\delta$ bounded.

However, it should be noted that the estimate in the last display also means that the native space of our radial functions is not a "small" space, in the sense that the weight function in the definition of its semi-norm does not increase fast. (In particular, the weight in the semi-norm $\|\cdot\|_{\phi_{\delta}}$ has no singularities since the Fourier transform, by which we divide, has no zeros, in contrast to the transforms of the radial functions in [19].)

\section{Spectral convergence orders}

Another point of interest is the result of letting $\lambda \rightarrow \infty$ in the construction of $\phi$ given in the penultimate section and not scaling. We contend that the radial function becomes an approximate identity, meaning its Fourier transform tends to a nonzero constant, whence the radial function, as a distribution in the real domain, to a multiple of the $\underline{\delta}$-distribution. As such, approximations with its translates by the centres on $h \mathbb{Z}^{n}$, say, can provide at a minimum local uniform convergence of best uniform approximations to continuous functions, when the "degree" $\lambda$ goes to infinity in tandem with $h \rightarrow 0$. The reason for this is that the $\underline{\delta}$-distribution is the identity operator with respect to convolution. Approximations from shifts of the radial function can then be viewed as a discretization of the approximand $f$ convolved with the radial function. In fact, we can even show a spectral convergence result for that continuous convolution, i.e., exponential convergence, the radial function still staying unscaled. For the reader's convenience, we outline the argument before embarking on the proof of the next theorem.

The key ingredient is that, when $\lambda \rightarrow \infty$, Bessel functions satisfy the convergence estimate

$$
\left|\frac{\sqrt{\pi}(2 \lambda+n)^{\lambda+n / 2+1 / 2}}{\exp (\lambda+n / 2)} J_{\lambda+n / 2}(t) t^{n / 2+1-\lambda}-t^{n+1}\right|=O\left(\frac{t^{n+1}}{2 \lambda+n}\right), \quad t>0,
$$

uniformly $\left[16\right.$, p. 225]. Still setting $g(\beta)=\left(1-\beta^{\mu}\right)_{+}^{\varphi}$, one concludes that the limiting expression of the suitably normalized Fourier transform $\hat{\phi}_{\lambda}$, where we here and subsequently denote its dependence on $\lambda$ by the index, is a multiple of

$$
r^{-n-2} \int_{0}^{\infty} t^{n+1}\left(1-t^{2 \mu} r^{-2 \mu}\right)_{+}^{Q} d t=\int_{0}^{\infty} t^{n+1}\left(1-t^{2 \mu}\right)_{+}^{Q} d t, \quad r \geqslant 0
$$

due to (4.1). This integral is constant and independent of $r$. Thus, remembering the 
work of Section 2, $\phi_{\lambda}$ must tend to a multiple of the $\underline{\delta}$-distribution. This statement is made precise in the following theorem, where the separation radius of $\left\{x_{j}\right\} \subset \mathbb{R}^{n}$ is $h=2 \sup _{x \in \mathbb{R}^{n}} \inf _{j}\left\|x-x_{j}\right\|_{\infty}$ which we assume to be positive and finite. Observe that this means there are infinitely many centres. The separation radius, e.g., for $h \mathbb{Z}^{n}$ is $h$.

Theorem 6. Every continuous function $f: \mathbb{R}^{n} \rightarrow \mathbb{R}$ can be arbitrarily closely, locally uniformly approximated by linear combinations of $\phi_{\lambda}\left(\cdot-x_{j}\right)$, if $h$ and $\lambda^{-1}$ are small enough, where $\phi_{\lambda}$ is the radial function of Theorem 1 and $h$ is the separation radius of the centres.

We immediately state another result that will follow from the proof of Theorem 6, where $*$ denotes convolution.

Corollary 7. Suppose $f \in L^{1}\left(\mathbb{R}^{n}\right) \cap C^{0}\left(\mathbb{R}^{n}\right)$ with $\mathbb{N} \ni v>n$ and that all of $f^{\prime}$ ' derivatives are integrable up to total order $v$. Then

$$
\left\|g_{\lambda} * f-f\right\|_{\infty}=O\left(\lambda^{-\gamma}\right), \quad \lambda \rightarrow \infty,
$$

where $\gamma$ is arbitrary and $g_{\lambda}$ is defined in (4.3).

Proof of Theorem 6. Let a $\gamma \in \mathbb{N}$ be given. Our first assertion is that there exists a

$$
g_{\lambda}=c_{\lambda}\left(1+d_{1}+d_{2}+\cdots+d_{\gamma-1}\right) \phi_{\lambda}
$$

with $c_{\lambda}, d_{i} \in \mathbb{R}$ so that for any $\tilde{f} \in L^{1}\left(\mathbb{R}^{n}\right)$ with integrable Fourier transform

$$
\left\|g_{\lambda} * \tilde{f}-\tilde{f}\right\|_{\infty}=O\left(\lambda^{-\gamma}\right)(=o(1)), \quad \lambda \rightarrow \infty .
$$

To prove this, we take Fourier transforms and estimate by Hölder's inequality

$$
\begin{aligned}
\left\|g_{\lambda} * \tilde{f}-\tilde{f}\right\|_{\infty} & \leqslant \frac{1}{(2 \pi)^{n}} \int_{\mathbf{R}^{n}}\left|\hat{g}_{\lambda}(t)-1 \| \widehat{\tilde{f}}(t)\right| d t \\
& \leqslant \frac{1}{(2 \pi)^{n}} \int_{\mathbf{R}^{n}}|\widehat{\tilde{f}}(t)| d t \sup _{t \in \mathbb{R}^{n}}\left|\hat{g}_{\lambda}(t)-1\right| .
\end{aligned}
$$

Now, according to [16, p. 225], (4.1) can be stated more precisely as

$$
J_{\lambda+n / 2}(t) t^{n / 2+1-\lambda}=\frac{t^{n+1} \exp (\lambda+n / 2)}{\sqrt{\pi}(2 \lambda+n)^{\lambda+n / 2+1 / 2}}\left(1+\frac{a_{1}}{\lambda+\frac{n}{2}}+\frac{a_{2}}{\left(\lambda+\frac{n}{2}\right)^{2}}+\cdots\right), \quad t>0,
$$

where $a_{i}$ are real coefficients. This, (4.2) and the penultimate display imply that (4.4) can be obtained by judicious, $\lambda$-dependant choice of each $d_{1}$, where $c_{\lambda}$ is a fixed constant multiple of $\exp (-\lambda-n / 2) \sqrt{\pi}(2 \lambda+n)^{\lambda+n / 2+1 / 2}$. Indeed 


$$
\begin{aligned}
\left|\hat{g}_{\lambda}(t)-1\right| & =\left|C c_{\lambda}\left(1+d_{1}+\cdots+d_{\gamma-1}\right) r^{-n-2} \int_{0}^{\infty} J_{\lambda+n / 2}(t) t^{n / 2-\lambda+1}\left(1-t^{2 \mu} r^{-2 \mu}\right)_{+}^{Q} d t-1\right| \\
& \leqslant C r^{-n-2} \int_{0}^{\infty} \frac{t^{n+1}}{\left(\lambda+\frac{n}{2}\right)^{\gamma}}\left(1-t^{2 \mu} r^{-2 \mu}\right)_{+}^{Q} d t \\
& =C \int_{0}^{\infty} \frac{t^{n+1}}{\left(\lambda+\frac{n}{2}\right)^{\gamma}}\left(1-t^{2 \mu}\right)_{+}^{Q} d t \\
& \leqslant C \lambda^{-\gamma}, \quad \lambda \rightarrow \infty,
\end{aligned}
$$

can be obtained. When $\gamma=3$, for instance, our choices for $d_{1}$ and $d_{2}$ are $-\frac{a_{1}}{\lambda+\frac{n}{2}}$ and $-\frac{a_{2}-a_{1}^{2}}{\left(\lambda+\frac{n}{2}\right)^{2}}$, respectively. This establishes (4.4).

Because $f$ can be locally uniformly approximated by $\tilde{f}$ with the required properties up to any desired accuracy, the theorem follows now from (4.4) through application of any suitable quadrature rule that uses the $x_{j}$ as points to evaluate the integrand to the (finite) integral $g_{\lambda} * \tilde{f}$.

Proof of Corollary 7. The same arguments as in the previous proof apply, except that we may directly take $f$ instead of $\tilde{f}$, because our assumptions imply, by a standard argument using integration by parts, $|\tilde{f}(x)|=O\left(\|x\|^{-n-1}\right)$ for $\|x\| \rightarrow \infty$, i.e., $\tilde{f} \in L^{1}\left(\mathbb{R}^{n}\right)$.

Remark. The convergence of the approximant from linear combinations of $x_{j}$-shifts of $\phi_{\lambda}$ can also be formulated in a distributional sense, using the notion of weak convergence. This is straightforward since $\phi_{\lambda}$ tends to the $\underline{\delta}$-distribution, and it is therefore not detailed here.

\section{REFERENCES}

1. M. ABRamowitz and I. STEGUN, Handbook of Mathematical Functions (Dover Publications, New York, 1972).

2. C. DE BOoR and A. RON, Fourier analysis of the approximation power of principal shift invariant spaces, Constr. Approx. 8 (1992), 427-462.

3. M. D. BuhmanN, Cardinal interpolation with radial basis functions: an integral transform approach, in Multivariate Approximation Theory IV (Walter Schempp, Karl Zeller, eds., International Series of Numerical Mathematics Vol. 90, Birkhäuser Verlag, Basel, 1989), 41-64.

4. M. D. BummanN, New developments in the theory of radial basis function interpolation, in Multivariate Approximation: From CAGD to Wavelets (K. Jetter and F. I. Utreras, eds., World Scientific, Singapore, 1993), 35-75.

5. M. D. BunmanN, Discrete least squares approximation and pre-wavelets from radial function spaces, Math. Proc. Cambridge Philos. Soc. 114 (1993), 533-558.

6. M. D. Bummann, Pre-Wavelets on Scattered Knots and from Radial Function Spaces: A Review, in Mathematics of Surfaces VI (G. Mullineux, ed., IMA Conference Proceedings Series, Oxford University Press, Oxford, 1996), 309-324. 
7. M. D. Buhmann and N. DYN, Spectral convergence of multiquadric interpolation, Proc. Edinburgh Math. Soc. 36 (1993), 319-333.

8. J. Duchon, Splines minimizing rotation invariant semi-norms in Sobolev spaces, in Constructive Theory of Functions of Several Variables (Walter Schempp, Karl Zeller, eds., LNM, Springer Verlag, Berlin, 1977), 85-100.

9. G. GaSPER, Positive integrals of Bessel functions, SIAM J. Math. Anal. 6 (1975), 868-881.

10. W. Golomb and H. F. WeInberger, Optimal approximation and error bounds, in Numerical Approximation (R. E. Langer, ed., University of Wisconsin Press, Madison, 1959), $117-190$.

11. C. A. Micchelli, Interpolation of scattered data: distance matrices and conditionally positive definite functions, Constr. Approx. 2 (1986), 11-22.

12. J. K. MisiewiCz and D. ST. P. Richards, Positivity of integrals of Bessel functions, SIAM J. Math. Anal. 25 (1994), 596-601.

13. R. SCHABACK, Multivariate interpolation and approximation by translates of a basis function, in Approximation Theory VIII (C. K. Chui and L. L. Schumaker, eds., World Scientific, Singapore, 1995).

14. R. SchabaCK and H. Wendland, Special cases of compactly supported radial basis functions, University of Göttingen, 1994, preprint.

15. E. M. STEIN and G. WeIss, Introduction to Fourier Analysis on Euclidean Spaces (Princeton University Press, Princeton, 1971).

16. G. N. Watson, A Treatise on the Theory of Bessel Functions (Cambridge University Press, Cambridge, 1952).

17. H. Wendland, Piecewise polynomial, positive definite and compactly supported radial functions of minimal degree, University of Göttingen, 1995, preprint.

18. R. E. Williamson, Multiply monotone functions and their Laplace transforms, Duke Math. J. 23 (1956), 189-207.

19. Z. WU, Multivariate compactly supported positive definite radial functions, University of Göttingen, 1994, preprint.

\author{
Mathematik Departement \\ ETH ZENTRUM \\ 8092 ZÜRICH \\ SWITZERLAND \\ E-mail address: mdb@math.ethz.ch \\ Current address: \\ Mathematik, LS8 \\ UNIVERSITÄT DORTMUND \\ D-44221 DORTMUND \\ GERMANY \\ E-mail address: mdb@math.uni-dortmund.de
}

